\title{
Acknowledgement to Reviewers of Social Sciences in 2018
}

Social Sciences Editorial Office

MDPI, St. Alban-Anlage 66, 4052 Basel, Switzerland

Published: 10 January 2019

Rigorous peer-review is the corner-stone of high-quality academic publishing. The editorial team greatly appreciates the reviewers who contributed their knowledge and expertise to the journal's editorial process over the past 12 months. In 2018, a total of 265 papers were published in the journal, with a median time to first decision of 27.03 days and a median time to publication of 48 days. The editors would like to express their sincere gratitude to the following reviewers for their cooperation and dedication in 2018:

Abbas, Azhar

Abu Shuleh, Mohammad

Aceleanu, Mirela

Adams, Michele

Addie, Jean-Paul

Adnan Raheel Shah, Syyed

Adusei-Asante, Kwadwo

Aggarwal, Aradhna

Agnich, Laura E.

Agrigoroaei, Stefan

Ahmed, Bipasha

Ahmed, Waqas

Ahn, Ji-Young

Ahuvia, Aaron

Aiken, Mike

Akkari, Abdeljalil

Akser, Murat

Aldikacti Marshall, Gul

Aleksić, Ana

Ali Nasir, Muhammad

Ali, Abbas

Ali, Sharafat

Alina, Haller

Alt, Rainer

Althouse, Ben

Amirkhanpour, Monaliz

Amsel, Eric

Ananda, Jayanath

Andorno, Roberto

André, Stéfanie

Antonopoulos, Georgios

Aparicio García, Marta Evelia
Arcuri, Sabrina

Armstrong, Robin

Asci, Serhat

Ashwood, Loka

Assari, Shervin

Åström, Berit

Atack, Iain

Atukeren, Erdal

Avent-Holt, Dustin

Avis, James

Axford, Nick

Aysa-Lastra, Maria

Ayuso, Luis

Azrael, Deborah

Bădescu, Gabriel

Badulescu, Alina

Bakaki, Zorzeta

Baldez, Lisa

Baldoli, Claudia

Baldwin, Debora R.

Bamford, Rebecca

Banerjee, Aparajita

Banerjee, Pallavi Amitava

Baran, Mette L.

Barcik, Agnieszka

Barker, Lecia

Barker, Valerie

Bar-Tal, Daniel

Bartkowski, John

Bartova, Alzbeta

Baser Ozturk, Bahar

Baunach, Dawn 
Baxi, Upendra

Beaulieu, Lionel "Bo"

Beazley, Harriot

Beck, Ashley

Becken, Susanne

Behnia, Behnam

Beier, Grischa

Belau, Linda

Belcher, John R.

Bell, David

Belloni, Milena

Berggren, Caroline

Bergsdóttir, Arndís

Berkowsky, Ronald

Bernardino, Jorge

Berntzen, Lasse

Berthold, Anne

Biehal, Nina

Bilecen, Basak

Biondo, Alessio Emanuele

Bix, Amy

Black, Leon

Blanchard Kyte, Sarah

Blizek, William L.

Block Jr., Ray

Blum, Sonja

Blumenthal-Barby, Martin

Bockarie, Abu

Bohm, Martha

Bonati, Sara

Bond, Bradley J.

Bondoc, Ionel

Boobbyer, Philip

Boonstra, Beitske

Borge-Diez, David

Bosworth, Kai

Bouma, Gary

Bowen, Mary Elizabeth

Bowman, Glenn

Bracken, David

Bradley, Harriet

Braithwaite, Charles

Breeze, Ruth

Brennan, Louis

Brezis, Elise S.

Brisman, Avi

Brock, Gregory

Brooks, Robert

Brown, James

Browne, Harry

Brutti, Zelda

Buchanan, Robert
Buchmann, Marlis

Buday, Amanda

Buelga, Sofia

Buente, Wayne

Bull, Glen

Burke, Jessica L.

Burnett, Jon

Burrows, Andrea C.

Byrd, Christy

Byrd, Derria

Byrne, Jennifer

Bywaters, Paul

Cai, Guoray

Camerino, Donatella

Camp, Gregory

Campbell, Heather

Campbell, Marilyn

Campillo Poza, Inés

Cannito, Maddalena

Canonico, Esther

Canosa, Antonia

Cant, Richard

Cantwell, Brendan

Caracciolo Di Torella, Eugenia

Carbonell Carrera, Carlos

Carlin, Eric

Carline, Anna

Carney, Terry

Caron, Rosemary

Carr, James

Carter, Angie

Cascajo, Rocio

Cassidy, John

Castro, Maria Pia

Cava, María J.

Cavaco, Carmen

Ceccato, Vania

Chacón, Jesús

Chang, Angela

Chapman, Andrew John

Chatalova, Lioudmila

Cheung, Li

Chiu, Yu-Ling

Choate, Peter W.

Choi, Myeonggil

Choo, Pei Ling

Choongo, Progress

Ciasullo, Maria Vincenza

Clapton, Gary

Clarke, Gerard

Classen, Audra

Clemans, Allie 
Clevenger, Aaron

Cockburn, Cynthia

Cognard-Black, Andrew J.

Collazo Expósito, Leslie Mahe

Collins, LaPorchia

Comes, Calin

Comfort, Louise

Conroy, Meredith

Contamin, Jean-Gabriel

Contreras, Diana

Cook, Amanda

Cook, Brian

Cooper, Simon

Cope, Michael

Cosentino, Antonietta

Cotton, Matthew

Coussens, Scott

Couturier, Yves

Coxhead, Ian

Crespi, Isabella

Crespo, Nuno

Croog, Rebecca

Crow, Bryan

Crow, Graham

Csiszár, Csaba

Ćulum, Bojana

Curci, Gabriele

Curry, Gwenetta

Cuthbert, Karen

Cuzán, Alfred G.

D’Acci, Luca

Dalla Rosa, Marco

Damiani, Paola

Dana, Léo-Paul

Dao, Loan

Darrow, Jessica

D'Auria, Anna

Davey, Judith

David, Isabel

Davies, Randall S.

Davis, Amy

Dayyala, Niharika

De Almeida, Claudia Ribeiro

De Boer, Jelle

De Boise, Sam

De Gruyter, Christopher

De Lacalle, Luis Norberto López

De Soysa, Indra

De Wit, Hans

Debebe, Gelaye

DeCarvalho, Lauren

Deepwell, Katy
Della Spina, Lucia

DelRosso, Jeana

DeMeritt, Jacqueline

Demirel-Pegg, Tijen

Dening, Tom

Devolder, Pierre

DeVotta, Neil

Dezetter, Anne

Diab, Ehab

Díaz Meneses, Gonzalo

Diaz, Vince

DiBenedetto, Maria K.

Diefendorf, Sarah

Diener, Ed F.

Diogo, Elisete

Do Céu Taveira, Maria

Doberneck, Diane

Doharty, Nadena

Dong, Qian

Donohue, William

Doucek, Petr

Dovì, Vincenzo

Down, Barry

Doyle, Stephanie

Dresden, Brooke E.

D'Souza, Natalia

$\mathrm{Du}$, Lin

Dundes, Lauren

Durkee, Myles

Durrant, Joan

Duvander, Ann-Zofie

Dykstra-DeVette, Tiffany A.

Dzuverovic, Nemanja

Edelsbrunner, Peter

Edwards, Mark

Ergene, Bogac

Eriksson, Lina

Eriksson, Tor

Espejo, Raul

Everitt, Julia

Everri, Marina

Falcone, Pasquale Marcello

Falk, Martin

Fawcett, Barbara

Fazio, John

Fekete, Mária Magdolna

Feng, Yang

Fernandez, Alberto

Ferreira, Marisa

Field, Laurie

Fields, Sarah K.

Fink, Arlene 
Finnegan, Fergal

Fiorentino, Michele

Fitton, Triona

Flanagan, Nina

Fleisher, Mark S.

Folgado, José Antonio

Fong, Eric

Fonseca, Luis Miguel Ciravegna

Fonte, Maria

Foody, Mairéad

Foreman, Anne M.

Forino, Giuseppe

Foster, Rachel E.

Fox, Stephen

Fox, Stuart

Fox, William E.

Franceschelli, Michela

Francesco, Duina

Freeze, Gregory

Fristedt, Sofi

Froehlich-Grobe, Katherine

Fusco, Giulio

Gagné, Isaac

Galeazzi, Gian Maria

Galella, Donatella

Ganau, Roberto

Gannon, Frédéric

Gao, Tao

Garcia, Amber

García, Diana

García, Jorge Luis

García, Marcelo V.

García-Martínez, Inmaculada

Garlick, Jeremy

Gaskell, George

Gasman, Marybeth

Gasparinatou, Alexandra

Gaston Gayles, Joy

Gavin, Helen

Gebhardt, Markus

George Mwangi, Chrystal

Geraint, Johnes

Gerbeti, Agime

Gibney, Raymond

Gidron, Noam

Gillespie, Brian

Gilmer, Mary Jo

Giuffrida, Nadia

Glynn, Peter J.

Goglio, Valentina

Gong, Xue

Gonzalez Canche, Manuel
González Valero, Gabriel

González, Andrés Vargas

Gonzalez-Benson, Odessa

González-López, Manuel

Gorard, Stephen

Górecka, Aleksandra

Gosetti, Giorgio

Grabara, Janusz

Granberry, Phillip J.

Gray, David

Green, Colin

Green, John J.

Griffiths, David

Grosse, Corrie

Grothe-Hammer, Michael

Grubaugh, Stephen G.

Grzegorczyk, Anna

Guarini, Enrico

Guina, Jeffrey

Gulino, Matteo

Gupta, Anna

Guthrie, Robert

Gutierrez, Antonio

Gutiérrez, Jose De Sola

Guzek, Dominika

Haas, Ingrid

Hailikari, Telle

Hajjar, Lisa

Hale, Lisa

Halvorsen, Beni

Han, The Anh

Hand, Caroline

Hanf, Jon

Hanifzadeh, Mohammadmatin

Hanlon, Bernadette

Harding, Simon

Harpin, Scott

Harris, Daniel I.

Harris, Richard

Hartung, Catherine

Hasebrook, Joachim Paul

Hassell, Hans J.G.

Hassler-Forest, Dan

Hélot, Christine

Henry, Lisa

Hentges, Beth

Hering, Amanda S.

Hernandez, Leandra

Herrera, Linda

Herreros, Francisco

Herrold, Catherine

Herteliu, Claudiu 
Herzig, Abbe H. Heyman, Gail

Hindman, Matt

Hine, Ben

Hobza, Vladimir

Hodbod, Jennifer

Hölbl, Marko

Hollin, Greg

Holm, Michael

Hong, Jun Sung

Howard, Simon

Howell, Aaron

Hsu, Hsun-Ta

$\mathrm{Hu}$, Tian

Huang, Xiaoyan

Huerta, Adrian H.

Hughes, Judy

Hughes, Nathan

Hung, Mei-Chuan

Hutchinson, Dawn

Huyser, Kimberly R.

Hyslop, Ian

Hytti, Ulla

Idiano, D'Adamo

Ihle, Andreas

Immelman, Aubrey

Irgens, Golnaz Arastoopour

Irimias, Anna

Iseppi, Luca

Isleem, Martin

Ismail, Tareq

Isopahkala-Bouret, Ulpukka

Ivan, Loredana

Ivascu, Larisa

Iyke, Bernard Njindan

Jachimowski, Kayla

Jackson, Richard

Jagger, Susan

Jagosh, Justin

James, Sherman A.

Jamil, Uzma

Jang, Sunghoon

Janousek, Christian

Janská, Eva

Jarvis, Rebecca M.

Jeljeli, Riadh

Jenlink, Patrick M.

Jennings, Laura

Jeske, Debora

Jha, Chandan

Jiang, Yan

Jianu, Iulia
Johnson, Amy

Johnson, Amy K.

Johnson, Derek

Jones, Steven

Jones, Tiffany

Jones-Eversley, Sharon

Jordan, Cathy

Joseph-Salisbury, Remi

Jump, Deborah

Jungbauer-Gans, Monika

Kafadar, Karen

Kahn, Shulamit

Kalimeri, Kyriaki

Kamen, Charles S.

Kanai, Akane

Kanavos, Andreas

Kang, Miliann

Kantono, Kevin

Karoń, Grzegorz

Katre, Aparna

Kavish, Daniel

Keating, Vincent Charles

Keddell, Emily

Keebler, Joseph

Kelman, Candice Carr

Kemp, Susan P.

Kendall-Taylor, Nathaniel

Kilkelly, Ursula

Kim, Eui Kyung

Kim, Hyun Woo

Kinderman, Daniel

Kirakosyan, Lyusyena

Kirby, Dianne

Klausen, Søren Harnow

Klein, Jennie

Klem, Bart

Kmec, Julie

Knight, Michelle

Knoester, Chris

Koech, Richard

Konstantinov, Vsevolod

Konzett, Delia Malia

Kort, Michael

Kossyvaki, Lila

Kostopoulos, Ioannis

Kouziokas, Georgios N.

Kovačić, Marko

Kramers, Anna

Kretzschmar, Andre

Krewani, Angela

Krienert, Jessie L.

Krupa, Olha 
Kubicek, Paul

Kucheva, Yana

Kurita, Takamitsu

Kurowska-Pysz, Joanna

Kushner Gadarian, Shana

Kyriacou, Chris

Kyriakopoulos, Grigorios L.

Labaree, David F.

Lajosi-Moore, Krisztina

Lajunen, Timo

Lakatos, Elena Simina

Lal, Kaushalesh

Lalueza, José Luis

Lambrechts, Wim

Lamin, Saffa

Lammi, Minna

Languilaire, Jean-Charles

Larsen, Peter Bille

Lass, Inga

Lasselle, Laurence

Laudal, Thomas

Lauerma, Hannu

Lebart, Ludovic

Lee, Ahlam

Lee, Ellie

Lee, Frances Lai Mui

Lee, Misook

Lee, Sang Yup

Lefevere, Jonas

Leibetseder, Bettina

Leicht, Kevin

Leitão, João Carlos Correia

Lenger, Alexander

Leon Rubio, Jose Maria

Leonard, Rosemary Jill

Leone, Luigi

Leone, Matthew C.

Lera, María José

Lerch, Julia C.

Lesclingand, Marie

Levac, Leah

Lever, John

Levesque, Lucie

Lewis, John

Li, Manyu

Liczmańska-Kopcewicz, Katarzyna

Lidén, Gustav

Lilenfeld, Lisa

Lillard, Colleen

Lima, Licínio C.

Lin, Tin-Chun

Lipinski, John
Lisinskienė, Aušra

Lloyd, Natalie

Lo Storto, Corrado

Loderer, Kristina

Lopez Jerez, Montserrat

López-López, Daniel

Lowery, Patrick

$\mathrm{Lu}, \mathrm{Yi}$

Lücking, Mirjam

Ludwig, Thomas

Luis Ubago, Jose

Luitel, Hari S.

Lundén, Janne

Luthra, Renee

Lyons, Tanya

Machimbarrena, Juan M.

Maciejewski, Grzegorz

Macioti, Maria Immacolata

Mackelprang, Romel

MacNell, Lillian

Macura, Dragana

Madsen, Wendy

Magaril, Elena

Magli, Francesca

Magrane, Diane

Mainar, Alfredo

Makarovič, Matej

Makuch, Karen E.

Malatzky, Christina

Malega, Ronald W.

Mallick, Bishawjit

Mansour, Jamal Ben

Marčeková, Radka

Marczak, Magdalena

Marin, Giovanni

Marine-Roig, Estela

Markvica, Karin

Martel, Andrew

Marti-Ballester, Carmen-Pilar

Martín Aragón, María Del Mar

Martínez-Ferrer, Belén

Mash, Holly B. Herberman

Maškarin Ribarić, Helga

Masters, Marick

Maturo, Antonio Francesco

Mayers, Raymond Sanchez

Mazurana, Dyan

McCaig, Colin

McCarthy, Jane

McClearn, Deedra

McCracken, Bonnie

McCrea, Rod 
McCullough, Laura

Mcdonald, Ted

McDowell, Charlie

McGill, Craig M.

McGinty, Emma

McKenzie, Grant

McQuillan, Julia

Mees, Heleen

Mei, Xiaohan

Melleuish, Greg

Melville, Angela

Menéndez, Ana Luisa Reyes

Merlock Jackson, Kathy Merlock

Merz, Johannes

Meszaros, John

Micera, Roberto

Michael, Bryane

Michaud, Gilbert

Michell, Dee

Middaugh, Ellen

Mifsud, Mark C.

Miha, Lesjak

Mikos, Lothar

Milczarek-Andrzejewska, Dominika

Miller, Catherine

Miller, Derek

Milligan, Richard

Millner, Edward

Mingboupha, Nathalie

Mirrlees, Tanner

Mishra, Atul

Miskolczi, Péter

Mitchell, Terry

Mocnik, Nena

Mollet, Tracey

Montgomery, LaTrice

Moorehead, Robert

Morada, Noel

Morciano, Daniele

Morea, Donato

Morozov, Boris

Morris, Kate

Morrison, Leanne

Morrissey, Anne

Moscatelli, Silvia

Moses, Gavriel

Mosley, Jacquelyn Dee

Mosselson, Jacqueline

Mróz, Anna

Mucina, Mandeep Kaur

Mujcic, Redzo

Mujtaba, Bahaudin
Mullins, Christopher W.

Mund, Marcus

Munoz-Guzman, Carolina

Munt, Sally

Muresan, Iulia Cristina

Murua, Imanol

Nackerud, Larry

Nam, Taewoo

Napal, María

Naples, Nancy

Napoli, Grazia

Nava, Luzma Fabiola

Navarre-Jackson, Layana

Nazir, Sajjad

Nelson, Laura K.

Neureiter, Michael

Newbold, Greg

Newton, Andrew

Nicolau, Juan Luis

Niemann-Lenz, Julia

Nikolaou, Ioannis

Nissen, Jayson

Nixon, Margaret

Njavro, Mario

Nobles, Matt

Nozawa, Shunsuke

Nunes, Sara

Nygren, Lennart

O'Leary, Kathleen

Oblad, Timothy

O'Brien, Kate

O'Dwyer, Jean

Offord, Baden

O'Kane, Eamonn

Olah, Judit

Olivas, Raul Navarro

Oncini, Filippo

Opdyke, Aaron

Orchard, Treena

Ormsbee, Floyd

Ortiz-Colón, Ana María

Osca, Amparo

Oshio, Atsushi

O'Toole, Mitchell

Owusu, Andrew

Oxendine, Alina

Paller, Jeffrey

Palmer, Erika

Palos-Sánchez, Pedro

Pan, Haozhi

Pan, Xi

Panagopoulos, Costas 
Panda, Bibhudutta

Paradies, Yin

Parisi, Mimmo

Park, Seunghyun

Parkes, Mitchell

Patel, Tina G.

Patel, Vibhuti N.

Paul, Christopher

Paulin, Alois

Pauly, Robert J.

Pavlovskaya, Marianna

Pawlowitsch, Christina

Pearson, Jamie

Peck, Nancy

Pedrini, Giulio

Pellerin Gammage, Marquita

Peretz, Tal H.

Perez, Victor W.

Peric, Marko

Perkins, Wendy

Perko, Igor

Perles-Ribes, José

Permanyer, Iñaki

Perrin, David

Perry, Barbara

Peters, Scott

Peterson, David A.M.

Petrjánošová, Magda

Pfeifer, Heather L.

Phillips, Coretta

Piehler, Kurt

Piggot, Georgia

Pirlott, Angela

Pluut, Helen

Poledrini, Simone

Popovac, Maša

Porczyński, Dominik

Posner, Paul W.

Poteyeva, Margarita

Powers, James S.

Prenzler, Tim

Privitera, Donatella

Psomopoulos, Constantinos S.

Ptak-Chmielewska, Aneta

Puertas Molero, Pilar

Pypłacz, Paula

Pyrialakou, Dimitra

Rada, Elena Cristina

Ragkos, Athanasios

Ramsey, John

Ramsland, Katherine

Randall, Yafiah Katherine
Rauch, Erwin

Rawwas, Mohammed

Reese, Gerhard

Reilly, Erin Dawna

Reimer, Thordis

Reinhardt, Jan D.

Reinke, Amanda J.

Rendleman, C. Matthew

Renold, Ursula

Requena, Félix

Ressler, Robert

Retallick, Michael

Revoredo Giha, Cesar

Ricci, Stefano

Riforgiate, Sarah

Rigg, Jonathan

Rincón, Blanca

Rivera Pinderhughes, Raquel

Roberts, Ken

Robinson, Grace

Roby, Jini

Rodríguez, Juan

Rodriguez, Nancy

Rodriguez-Modroño, Paula

Rogerson, Michelle

Romer, Dan

Romero, Luis Miguel

Ronzi, Sara

Rosen, Rachel

Rosser, Sue V.

Rostam-Afschar, Davud

Roubík, Hynek

Rowland, Emma

Rowland, Tracey

Ruban, Dmitry

Rueger, Bernhard

Ruggieri, Ruggero Andrisano

Ruhle, Sascha

Ryabov, Igor

Sádaba, Charo

Salazar, Leslie Ramos

Salinas Jr., Cristobal

Salmerón, Esther

Salvatore, Rita

Salvioni, Cristina

Samadi, Sayyed Ali

Sánchez Rojo, Alberto

Sanchez, Benito

Sandy, Larissa

Santos Silva, Dora

Sarathchandra, Dilshani

Saunders, Rebecca 
Saura, Jose Ramon Sawrikar, Pooja

Sayas, John

Scandurra, Cristiano

Scaramuzzino, Roberto

Schapper, Andrea

Scherer, Lexie

Schierenbeck, Isabell

Schmidt, Klaus

Schmitz, Rachel

Scholz, Johannes

Schulz, Michael

Schwingel, Andiara

Seiz Puyuelo, Marta

Sell, Tara

Serafini, Gianluca

Serpa, Sandro

Serrat, Rodrigo

Service, Brenda

Servidio, Rocco

Shakya, Kabindra Man

Sharafizad, Jalleh

Sharma, Isha

Shea, Jennifer M.

Sheridan, Lorraine

Shi, Wei

Shifflet-Chila, Erica D.

Shojafar, Mohammad

Siakwah, Pius

Sidebotham, Peter

Sim, Seung-Gyu

Simeon, James C.

Simos, Joanna

Sinanan, Jolynna

Sinclair, Amanda

Sinclair, Christine

Sinha, Vandna

Sipiläinen, Timo

Skattebol, Jennifer

Slay, Kelly E.

Slusky, David

Smith, Kimberley J.

Smith-Orr, Courtney

Smolacg, Klaudia

Soanes-White, Tammy

Sobocińska, Magdalena

Solomon, Offiong Helen

Song, Keng-hie

Song, Lily

Soo, Nikki

Spencer, Leland G.

Spillare, Stefano
Spyrou, Spyros

Squires, Peter

Srour, Anan

Staines, Jo

Ștefănescu-Mihăilă, Ramona Olivia

Štefko, Róbert

Steinberg, Gerald

Steirer, Greg

Stevens, Dan

Stevenson, Jacqueline

Stewart, Felicia

Stewart, William

Stich, Amy

Stonard, Karlie

Stone, Cathy

Stough, Roger

Strong, Catherine

Sturman, Kathryn

$\mathrm{Su}, \mathrm{Ye}$

Suarez, Carla

Sue, Derald

Suhaili Ramli, Nur

Sumerau, J. E.

Sundin, Ebba

Sung, Jong-Sang

Sygit, Katarzyan

Sylwester, Kevin

Szczepankiewicz, Elżbieta

Szczepańska-Woszczyna, Katarzyna

Sznycer, Daniel

Szulc, Lukasz

Taeihagh, Araz

Tampio, Nicholas

Tamura, Robert

Tang, Leilei

Taylor, Sandra

Taysum, Alison

Teachman, Gail

Teixeira, Carlos

Tellidis, Ioannis

Ter Horst, Eleanor

Terrazas Angulo, German

Testa, Riccardo

Thai, Yvonne

Thimm, Viola

Tholen, Gerbrand

Thomas, Talya D.

Thompson, Carol

Thornley, Peter

Thoron, Andrew

Thurairajah, Kalyani

Tiller, Rachel 
Timberlake, Jeffrey

Tong, Fan

Topa, Gabriela

Torgersen, Eivind Nessa

Torres-Reveron, Annelyn

Tragaki, Alexandra

Tremolada, Marta

Trier-Bieniek, Adrienne

Truong, Long

Tsai, Beth

Tseloni, Andromachi

Tsikouras, Panagiotis

Tuckett, Alan

Tuncdogan, Aybars

Turner, Lynn H.

Urban, Mathias

Urban, Susanne

Valero, Magali

Van Tuijl, Erwin

Vasseur, Liette

Vaughan, Kenneth

Vázquez Burguete, Jose Luis

Veen, Esther

Veiga, Ivo

Vera Gray, Fiona

Vermeulen, Floris

Veugen, Connie

Vignoli, Daniele

Villarreal, Brandilynn

Villis, Tom

Visvizi, Anna

Vloeberghs, Ward

Vogt, Sonja

Von Keyserlingk, Luise

Wakeling, Paul

Walker, Marvin

Waller, Richard

Walsh, Christine

Walsh, Moira

Wang, Jieh-Jiuh

Wang, Ruoniu

Wang, Yang

Wang, Yanling

Wang, Yuanqiong

Watkinson, Ailsa M.

Waygood, Edward Owen D.

Webb, Lynne M.

Weber, Mark

Weedon, Elisabet

Wegmann, Jake

Weiler, Stephan

Weiner, Adele
Weisgram, Erica

Weismayer, Christian

Weiss, Herbert F.

Weller, Sally

Westlund, Oscar

Wettstein, Markus

Wetzel, Dominic

Wharton, Amy

Wheeler, Stephen

Whelan, Eoin

White, Kate

Whitford, Denise K.

Whitley, Elise

Wiersma, Beth

Wilkes, Rima

Wilkinson, Matthew L. N.

Williams, Joah L.

Williams, Lucy

Williamson, Ryan D.

Wiseman, Angela

Wolbring, Tobias

Wolff, Nancy

Woodard, Ryan

Woods, Simon

Wozniak, Kathryn

Wright, Amy

$\mathrm{Wu}$, Jo

$\mathrm{Wu}, \mathrm{Qi}$

$\mathrm{Wu}$, Stephen

Wurtele, Sandy K.

Wyer, Mary B.

Wyile, Andrea Schwenke

Wyness, Gillian

Wyver, Shirley

$\mathrm{Xu}$, Lei

Yager, Zali

Yasmeen, Samina

Yeung, King-To

Yilmaz, Kuzey

Yoon, Sung Man

Yousefzadeh, Sepideh

Yu, Han

Zaborskis, Apolinaras

Zadra, Cinzia

Zainuddin, Nadia

Zajda, Joseph

Zhai, Qingguo

Zhao, Xiaoai

Zhu, Lin

Zhuang, Jun

Zierahn, Ulrich

Zitcer, Andrew 
Živanović, Predrag

Žostautiené, Daiva
Zscheischler, Jana

Zuccoli, Franca

(). (1)

(C) 2019 by the authors. Licensee MDPI, Basel, Switzerland. This article is an open access article distributed under the terms and conditions of the Creative Commons Attribution (CC BY) license (http://creativecommons.org/licenses/by/4.0/). 